\title{
Control intraoperatory glucose levels with modified atlanta scheme in subtotal partial hepatectomy: Case
} report

\begin{abstract}
We report the case of an 84-year-old patient with diagnosis of colon cancer with liver metastases in segment six. The patient required partial subtotal right hepatectomy. Glycemic control in patients undergoing liver surgery is vital, due to the complexity and secondary metabolic repercussions that arise from surgical stress. The dysregulation of glucose metabolism is associated with greater morbidity and mortality in these patients. In our patient, glucose levels were controlled based on the modified Atlanta scheme. There were no variations during the transanesthetic or hypoglycaemic events. The modified Atlanta scheme provides adequate efficacy and safety for the metabolic control of glucose in patients undergoing liver surgery.
\end{abstract}

Keywords: Blood Glucose, Glucose Metabolism, Glucose Dysregulation, Hepatectomy

\author{
Case Report \\ Volume I0 Issue I - 2018 \\ Cuevas-García J,' Quintas-Acevedo M,' \\ Velásquez-Paz AL, ${ }^{2}$ Navarro-Hernández QC, ${ }^{2}$ \\ Trápaga-Millares $\mathrm{Y}^{\prime}$ \\ 'Department of Anesthesiology, México \\ ${ }^{2}$ Department of Planning, Teaching and Research, México
}

Correspondence: Jesús Cuevas García, Department of Anesthesiology, Aldama Street, number less, San Bartolo Coyotepec, The Regional High Specialty Hospital of Oaxaca, Oaxaca, México, CP 71256, Tel 52 (I) 951 171922I, Email drcueg@outlook.com

Received: February 20, 2018 | Published: February 22, 2018
Abbreviations: ASA, Society of Anesthesiologists; RAQ, Surgical Anesthetic Risk; OR, Odds Ratio; ADA, American Diabetes Association; ICU, Intensive Care Unit; ECOG, Eastern Cooperative Oncology Group; MAC, Minimum Alveolar Concentration; PEEP, Positive End-Expiratory Pressure.

\section{Introduction}

The control of glucose as a metabolic factor is of great importance in patients undergoing hepatectomy, due to the level of complexity and the metabolic repercussions secondary to the surgical stress that are generated. This dysregulation of glucose metabolism is associated with perioperative complications, increased morbidity and mortality of the patient subjected to this type of intervention. Atlanta scheme is not only easy to reproduce, with a quick response to the infusion change, but also has high levels of safety with reports of up to $0.6 \%$ of hypoglycaemic events. It is a tool with a high level of efficiency in operating rooms and intensive therapy.

\section{Case presentation}

Male 84 years old, with a history of type 2 diabetes mellitus of seven years evolution, treated with metformin, and prostatectomy 7 years ago; in addition to irritable bowel syndrome, for which diagnostic colonoscopy was performed in 2012. We found poorly differentiated adenocarcinoma colon, treated with laparoscopic hemicolectomy and chemotherapy. In 2016, liver metastases were detected by primary colon.

The patient was scheduled to perform partial subtotal right hepatectomy. The pre-anesthetic evaluation was: ASA II, RAQ EII-B, KARNOSFKY $80 \%$ ECOG 1. Anesthetic technique started with previous anxiolysis with midazolam $30 \mathrm{mcg} / \mathrm{kg}$; in addition to peridural block at level L1-L2 with permeable inert peridural catheter, previous oxygenation, anesthetic induction with propofol $2 \mathrm{mg} / \mathrm{kg}$, narcosis with fentanyl $4 \mathrm{mcg} / \mathrm{kg}$, cisatracurium $0.15 \mathrm{mg} / \mathrm{kg}$, lidocaine $1 \mathrm{mg} / \mathrm{kg}$. Intubation is performed with direct laryngoscopy, maintained with mechanical ventilation controlled by volume (running volume $6 \mathrm{ml} / \mathrm{kg}$, respiratory rate 15 , positive pressure at the end of expiration $5 \mathrm{cmH}_{2} 0$, inspired fraction of oxygen $50-60 \%$ ). Invasive monitoring: central venous pressure $3-5 \mathrm{cmH}_{2} 0$, invasive blood pressure 50 $60 \mathrm{mmHg}$. Maintenance: nitroglycerin infusion dose $0.5-1 \mathrm{mcg} / \mathrm{kg} /$ min, fentanyl in plasma concentration $6 \mathrm{mg} / \mathrm{ml}$. Sevoflorano minimum alveolar concentration MAC $0.8-1$, ropivacaine infused at a $0.375 \%$ concentration via epidural. Modified Atlanta infusion scheme was prepared with regular rapid-acting insulin 50UI dilution in $50 \mathrm{ml}$ of $0.9 \%$ saline solution, blood glucose measurements were taken every hour during the procedure. Start of infusion of insulin with pump (Baxter colleague volumetric Infusion ${ }^{\mathrm{TM}}$ ) $0.5 \mathrm{ml} /$ hour with basal glycemia $84 \mathrm{mg} / \mathrm{dl}$ by gasometry (GEM equipment, Premier $3000^{\mathrm{TM}}$ ), second dose was of $105 \mathrm{mg} / \mathrm{dl}$ modified perfusion 4 rows up, with a volume of $1.3 \mathrm{ml} /$ hour, third takes $102 \mathrm{mg} / \mathrm{dl}$, continuous with scheme at $1.3 \mathrm{ml} /$ hour, fourth takes $120 \mathrm{mg} / \mathrm{dl}$ progresses to $1.8 \mathrm{ml} /$ hour. The patient withdraws from the extubation room, hemodynamically stable, with total bleeding of $700 \mathrm{ml}$. She was admitted to the intermediate care unit with capillary glycemia of $140 \mathrm{mg} / \mathrm{dl}$ and central glucose of $114 \mathrm{mg} / \mathrm{dl}$ (Table 1).

Table I Glucose variations during the procedure

\begin{tabular}{ll}
\hline Glucose values & \\
\hline Initial blood collection & $84 \mathrm{mg} / \mathrm{dl}$ \\
Second hour & $105 \mathrm{mg} / \mathrm{dl}$ \\
Third hour & $102 \mathrm{mg} / \mathrm{dl}$ \\
fourth hour & $120 \mathrm{mg} / \mathrm{dl}$ \\
Admission to $I C U$ & $114 \mathrm{mg} / \mathrm{dl}$ \\
\hline
\end{tabular}




\section{Discussion}

Hepatectomy great importance in the treatment of liver cancer. Anesthetic management ranges from hemodynamic control management of postoperative pain; anesthetic technique will always depend on the condition of the patient, his hepatic and hemodynamic function, and his nutritional status. ${ }^{1}$ The indications for surgery in $52 \%$ of cases are due to metastatic disease, followed by primary liver tumors with $16 \%$ and, thirdly, for malignancy in the bile ducts with $10 \% .^{2}$ In our case, indication for surgery was metastasis. Hepatectomy is associated with multiple disorders of metabolism, specifically glucose; being a risk factor for renal failure, infections of the surgical site and higher incidence of sepsis. It has been reported that hyperglycemia increases the inflammatory state, with increased cytokines such as interleukin- 6 , tumor necrosis factor- $\alpha$, and interleukin-18. An important point during hepatectomy are pringle events or total vascular isolations; what is sought with these maneuvers is to reduce the amount of bleeding during the moment of resection, since irrigation is important and a central point to consider in this surgical event. These maneuvers generate glucose peaks due to the transition of the glycogen decomposition within hepatocytes secondary hypoxia that occurs in each pringle..$^{3-5}$

In addition to this, due to insulin resistance induced by liver disease per se, glucose metabolism is aggravated by hepatectomy and is not reversed during the intraoperative neohepatic stage; functional liver function then plays a decisive role in the metabolism of glucose. ${ }^{6}$ In this case, the patient had a history of diabetes mellitus, requiring a more efficient control of glycemia. Few interventions, such as the use intensive insulin regimen, improved the survival of critical patients, preventing the onset of acute kidney injury, fewer transfusions, helping to improve erythropoiesis or reducing hemolysis. ${ }^{7}$ Insulin therapy reduces insulin resistance related to trauma, increases glycogen reserves, provides an anti-inflammatory effect and improves the defense of the immune system against infection, in addition to improving mitochondrial integrity with various clinical benefits. ${ }^{8-10}$

The insulin infusion algorithms that have shown the greatest benefits are those with the following characteristics for greater effectiveness; the first is that they are designed for dose-response; second, that there be monitoring capacity in the perioperative period or in intensive therapy, and third, perhaps the most important, is the stability and response capacity of the infusion; since an infusion can be so aggressive that it leads to hypoglycemia, or on the contrary expose the patient to prolonged hyperglycemia, significantly reducing in-hospital mortality by $16 \%$, OR of $0.84 .{ }^{11}$ Modified Atlanta scheme used in our patient, and meets these three fundamental characteristics. Krinsley $\mathrm{J}$ found that mortality decreased by $29.3 \%$, finding a greater benefit from intensive insulin therapy in patients with septic shock, with neurological and surgical disease. ${ }^{12}$ The American Diabetes Association (ADA) recommends the initiation of insulin infusion with values $180 \mathrm{mg} / \mathrm{dl}$, however, there is a consensus to use more stringent values between $110-140 \mathrm{mg} / \mathrm{dl}$ if there is a low rate of documented hypoglycaemia, some authors have described greater morbidity with these values due to hypoglycaemic events, however not in the surgical population. ${ }^{13,14}$ Griesdale D et al. ${ }^{15}$ conducted a meta-analysis that included the NICE-SUGAR study demonstrating that patients undergoing scheduled surgical events may have greater benefit derived from insulin therapy with stricter glucose controls. ${ }^{15}$ There are several insulin protocols, such as those by Markovitz et al and
Davidson et al. The latter occupies the formula: insulin dose $/ \mathrm{h}=(\mathrm{blood}$ glucose in mg/dl-1-60) x multiplier. This dynamic algorithm, also called modified Atlanta, is presented in tabular form, with rows that represent ranges of blood glucose and columns that represent insulin doses based on patients insulin sensitivity, representing individualized control according sensitivity of each patient. When the glucose is above $110 \mathrm{~m} / \mathrm{dl}$, the insulin dose increases by moving one column to the right, when the blood glucose level decreases in at least one range, but it is still above $110 \mathrm{mg} / \mathrm{dL}$, the insulin dose decreases within the same column, the lower limit of safety zone is $85 \mathrm{mg} / \mathrm{dl}$, this allows to reduce the dose to a blood glucose of $84 \mathrm{mg} / \mathrm{dl}$ to reduce the risk of hypoglycemia, once the goal of blood glucose between 85 and 110 $\mathrm{mg} / \mathrm{dL}$, green area (Figure 1), insulin is kept in the same column. Should be mentioned that with this protocol, hypoglycemia events have been reported in ranges from $0.6 \%$ to $2.6 \%$, and with lower need for insulin use in the ICU in the postsurgical unit. ${ }^{16-18}$ During procedure hypoglycemia events did not occur at any time, was not necessary to use dextrose in the procedure, which confers not only effectiveness but also safety that is one determining points when choosing infusion scheme insulin.

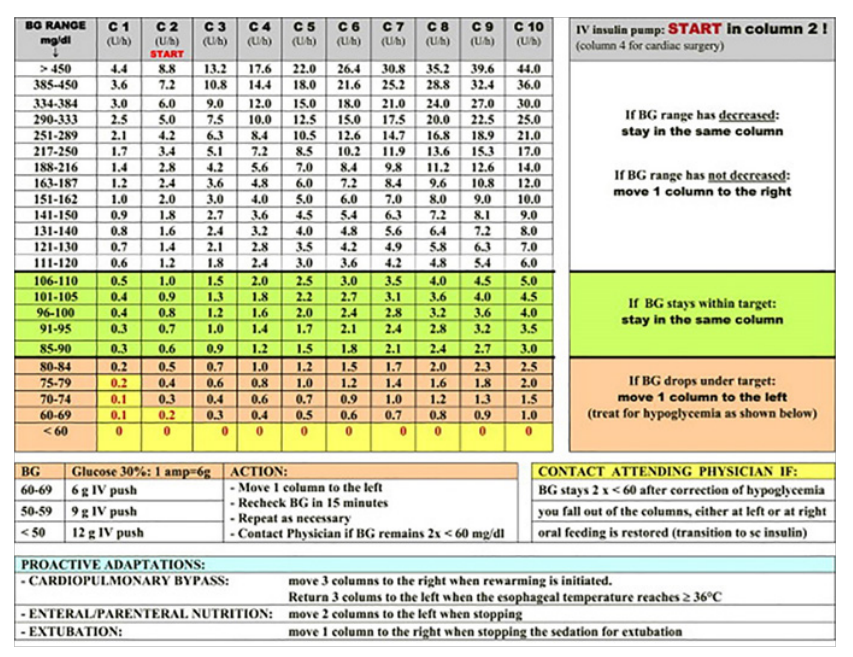

Figure I Protocol for continuous iv insulin administration for BG target 80-1 10 mg/dl (O.L.V. hospital Aalst, Belgium, 2009). (300 dpi).

\section{BG, blood glucose; C, column}

In this case report, an infusion of $0.5 \mathrm{ml} /$ hour was started due basal glucose that was reported by gasometry of $84 \mathrm{mg} / \mathrm{dl}$, important item was the very small variability between the 4 doses during the procedure, generating great benefit, since the variability of glucose linked to the complications mentioned above. The patient's discharge to ICU with central glucose values of $112 \mathrm{mg} / \mathrm{dl}$ allowed for better control from the start in the postoperative period and greater advantages for a quick and better recovery.

\section{Acknowledgments}

We thank the doctors of the Anesthesiology Service who will help in the writing of this article.

\section{Conflict of interest}

The author and the co-authors declare no conflict of interest. 


\section{References}

1. Cuéllar-Guzmán L,Pérez-GonzálezÓ. Consideraciones perioperatorias en el paciente oncológico sometido a hepatectomía. GAMO. 2016;15(6):358365 .

2. Dimick J. Hepatic Resection in the United States. Arch Surg. 2003;138(2):185.

3. Restoux A, Paugam-Burtz C. Anestesia-reanimación para la cirugía hepática y el trasplante hepático. EMC - Anestesia-Reanimación. 2015;41(2):1-22.

4. Mita N, Kawahito S, Soga T, et al. Strict blood glucose control by an artificial endocrine pancreas during hepatectomy may prevent postoperative acute kidney injury. J Artif Organs. 2016;20(1):76-83.

5. Maeda H, Okabayashi T, Nishimori I, et al. Hyperglycemia during hepatic resection: continuous monitoring of blood glucose concentration. Am J Surg. 2010;199(1):8-13.

6. Shangraw R, Hexem J. Glucose and potassium metabolic responses to insulin during liver transplantation. Liver Transpl Surg. 1996;2(6):443454.

7. van den Berghe $G$, Wouters $P$, Weekers $F$, et al. Intensive Insulin Therapy in Critically Ill Patients. N Engl J Med. 2001;345(19):1359-1367.

8. Fisette A, Hassanain M, Metrakos P, et al. High-Dose Insulin Therapy Reduces Postoperative Liver Dysfunction and Complications in Liver Resection Patients through Reduced Apoptosis and Altered Inflammation. J Clin Endocrinol. 2012; 97(1):217-226.

9. Wrighton LJ, O’Bosky KR, Namm JP, et al. Postoperative management after hepatic resection. J Gastrointest Oncol. 2012;3(1):41-47.
10. Vanhorebeek I, De Vos R, Mesotten D, et al. Protection of hepatocyte mitochondrial ultrastructure and function by strict blood glucose control with insulin in critically ill patients. Lancet. 2005;365(9453):53-59.

11. Bode BW, Braithwaite SS, Steed RD, et al. Intravenous insulin infusion therapy: indications, methods, and transition to subcutaneous insulin therapy. Endocr Pract. 2004;10 Suppl 2:71-80.

12. Krinsley J. Effect of an Intensive Glucose Management Protocol on the Mortality of Critically Ill Adult Patients. Mayo Clin Proc. 2004;79(8):992-1000.

13. Jacobi J, Bircher N, Krinsley J, et al. Guidelines for the use of an insulin infusion for the management of hyperglycemia in critically ill patients. Crit Care Med. 2012;40(12):3251-3276.

14. Finfer S, Chittock D, Li Y, et al. Intensive versus Conventional Glucose Control in Critically Ill Patients. N Engl J Med. 2009;360(13):1283-1297.

15. Griesdale DE, de Souza RJ, van Dam RM, et al. Intensive insulin therapy and mortality among critically ill patients: a meta-analysis including NICE-SUGAR study data. CMAJ. 2009;180(8):821-827.

16. Nobels F, Lecomte P, Deprez N, et al. Tight glycaemic control: clinical implementation of protocols. Best Practice \& Research Clinical Anaesthesiology. 2009;23(4):461-472.

17. Lecomte P, Foubert L, Nobels F, et al. Dynamic Tight Glycemic Control During and After Cardiac Surgery Is Effective, Feasible, and Safe. Anesth Analg. 2008;107(1):51-58.

18. Cammu G, Vermeiren K, Lecomte P, et al. Perioperative blood glucose management in patients undergoing tumor hepatectomy. Journal Of Clinical Anesthesia. 2009;21(5):329-335. 